\title{
Preservação da fertilidade e quimioterapia: uma revisão integrativa
}

\author{
Fertility preservation and chemotherapy: an integrative review \\ Preservación de la fertilidad y quimioterapia: una revisión integrativa
}

Ana Clara Veloso de Assis ${ }^{1 *}$, Danielle Mendonça Jatobá1, Guilherme Brasil Maia², Raissa Rego de Araujo ${ }^{1}$, Sheyla Mendes de Sousa ${ }^{1}$, Brenda Tayrine Tavares Souza ${ }^{1}$, Albênica Paulino dos Santos Bontempo'.

\section{RESUMO}

Objetivo: Observar questões relacionadas à preservação da fertilidade de mulheres e o tratamento quimioterápico por meio de uma revisão integrativa. Métodos: Revisão integrativa realizada na Biblioteca Virtual em Saúde com os descritores "Enfermagem", "Fertilidade" e "Tratamento Farmacológico" publicados nos últimos 5 anos. A seleção procedeu em 3 etapas: leitura do título, resumo e leitura na íntegra. Para análise estatística utilizou-se o método PRISMA. Resultados: Foram localizados 286 artigos. Removidos os duplicados e indisponíveis, em associação com filtros e aplicação dos critérios de elegibilidade, sobraram 20 artigos. Observou-se aumento da taxa de sobrevivência a longo prazo de pacientes com câncer e da demanda por preservação da fertilidade para possibilitar a maternidade. Estudos mostram o valor da prática de enfermagem, exigindo habilidades para lidar com os sentimentos dos outros e com as suas próprias emoções frente ao cuidado oncológico. Considerações finais: Os tratamentos para o câncer aumentam o risco de infertilidade de mulheres em idade reprodutiva. A Preservação da fertilidade aumenta a possibilidade de as sobreviventes se tornarem mães. O tratamento requer abordagem multidisciplinar, com destaque para equipe de enfermagem no que diz respeito ao apoio, aconselhamento e educação durante o tratamento.

Palavras-chave: Fertilidade, Tratamento farmacológico, Enfermagem.

\section{ABSTRACT}

Objective: Observe issues related to the preservation of fertility in women and chemotherapy treatment through an integrative review. Methods: Integrative review carried out in the Virtual Health Library with the descriptors "Nursing", "Fertility" and "Drug Therapy" published in the last 5 years. The selection proceeded in 3 steps: title reading, abstract and full reading. For statistical analysis, the PRISMA method was used. Results: 286 articles were found. After removing the duplicates and unavailable, in association with filters and application of the eligibility criteria, 20 articles remained. There was an increase in the long-term survival rate of cancer patients and in the demand for fertility preservation to enable motherhood. Studies show the value of nursing practice, demanding skills to deal with the feelings of others and their own emotions in cancer care. Final considerations: Cancer treatments increase the risk of infertility for women of childbearing age. Fertility preservation increases the chance of survivors becoming mothers. Treatment requires a multidisciplinary approach, with emphasis on the nursing team with regard to support, counseling and education during treatment.

Key words: Fertility, Drug therapy, Nursing.

\section{RESUMEN}

Objetivo: Observe cuestiones relacionadas con la preservación de la fertilidad en las mujeres y el tratamiento de quimioterapia a través de una revisión integradora. Métodos: Revisión integrativa realizada en la Biblioteca Virtual en Salud con los descriptores "Enfermería", "Fertilidad" y "Tratamiento farmacológico" publicado en los últimos 5 años. La selección se realizó en 3 pasos: lectura del título, resumen y lectura completa. Para el análisis estadístico se utilizó el método PRISMA. Resultados: Se encontraron 286 artículos. Después de eliminar los duplicados y no disponibles, en asociación con los filtros y aplicar los criterios de elegibilidad, quedaban 20 artículos. Hubo un aumento en la tasa de supervivencia a largo plazo de los pacientes con cáncer y en la demanda de preservación de la fertilidad para permitir la maternidad. Los estudios demuestran el valor de la práctica de la enfermería, exigiendo habilidades para lidiar con los cuidados del cáncer. Consideraciones finales: Los tratamientos contra el cáncer aumentan el riesgo de infertilidad. La preservación de la fertilidad aumenta las posibilidades de que las sobrevivientes se conviertan en madres. El tratamiento requiere un enfoque multidisciplinario, con énfasis en el equipo de enfermería en lo que respecta al apoyo, asesoramiento y educación durante el tratamiento.

Palabras clave: Fertilidad, Tratamiento farmacológico, Enfermería.

${ }^{1}$ Centro Universitário (UDF), Brasília - DF. *E-mail: anaclara.0512@hotmail.com

2 Universidade de Brasília (UnB), Brasília - DF. 


\section{INTRODUÇÃO}

A quimioterapia, de acordo com o Instituto Nacional de Câncer (INCA), é uma das formas de tratamento sistêmico do câncer que utiliza medicamentos denominados genericamente como "quimioterápicos". Seu mecanismo de ação impede a proliferação por meio da lise das células cancerígenas (INCA, 2021). Entretanto, esse tratamento tem um impacto significante na fertilidade, visto que as diferentes drogas encontradas na quimioterapia, apresentam vários graus de toxicidade, o que aumenta o risco de afetar órgãos reprodutores, podendo levar à amenorreia transitória, falência ovariana e a infertilidade. A infertilidade pode impactar de forma negativa os planos reprodutivos da mulher e/ou casal que deseja uma gravidez futura ou ainda não tem prole constituída (SILVA S, et al., 2021).

A oncofertilidade, que é o campo médico que une a oncologia e a reprodução, oferece aos profissionais de saúde e aos pacientes a oportunidade de otimizar a fertilidade residual (ASHIZAWA M e KANDA Y, 2020). Tem como objetivo principal aumentar o acesso dos pacientes com câncer ao aconselhamento sobre fertilidade e a sua preservação com o intuito de melhorar a qualidade de vida geral dos sobreviventes (RASHEDI AS, et al., 2020).

Em todo o mundo, cerca de 1,7 milhão de meninas e mulheres com menos de 50 anos são diagnosticadas com câncer anualmente, $5 \%$ afetam jovens com idade aproximada de 35 anos, ou seja, no seu auge reprodutivo. Devido aos avanços no tratamento, houve uma melhoria na expectativa de vida, resultando em uma população crescente de sobreviventes. No entanto, esses tratamentos podem afetar a função ovariana, causando insuficiência puberal, infertilidade e Insuficiência Ovariana Prematura (IOP). Assim, é possível afirmar que o comprometimento da função reprodutiva é o efeito colateral mais prevalente do tratamento anticâncer a longo prazo (JAYASINGHE YL, et al., 2018). Geralmente, mulheres reprodutivamente ativas possuem o armazenamento de óvulos baixo e a probabilidade de o tratamento ocasionar uma queda drástica da reserva ovariana é alta (ROO SF, et al., 2017).

Ainda segundo o INCA, em 2020, o Brasil alcançou o número de 316.280 novos casos de neoplasias de um modo geral. A distribuição dos diferentes tipos de câncer sugere uma alta transição epidemiológica em andamento (SILVA L, et al., 2019). A distribuição da incidência de câncer mostra que a região sudeste concentra mais de $60 \%$, seguida pela região nordeste $(27,8 \%)$ e sul $(23,4 \%)$ (INCA, 2021).

O cuidado com a saúde reprodutiva da mulher em tratamento de câncer é multiprofissional, e de acordo com o Decreto no 94.406/1987, o enfermeiro é um dos profissionais integrantes da equipe de saúde na atenção obstétrica e reprodutiva (QUEIROZ ABA, et al., 2020). A enfermagem faz-se de grande importância durante todo $o$ tratamento quimioterápico, sendo esses os profissionais que acompanham continuamente 0 paciente e que fornecem diversas orientações relacionadas ao tratamento e educação em saúde sobre a preservação da fertilidade a todos os envolvidos. Esta orientação é essencial na compreensão destes pacientes e familiares, sendo possível sanar todas as dúvidas e possibilitar uma melhor aderência ao tratamento quimioterápico e no enfrentamento diante da patologia (KEIM-MALPASS J, et al., 2017; OLIVEIRA FPB e GOMES KR, 2019).

A implementação dos cuidados ao paciente com câncer exige do enfermeiro a pluralidade de conhecimento e flexibilidade na atuação e, quando estão associadas a uma boa sistematização da assistência, as ações de enfermagem ao paciente são determinantes para uma gerência de boa qualificação (CARMO R, et al., 2019).

Diante do exposto, este artigo teve por objetivo observar questões relacionadas à preservação da fertilidade de mulheres e o tratamento quimioterápico por meio de uma revisão integrativa.

\section{MÉTODOS}

Trata-se de uma revisão integrativa realizada na Biblioteca Virtual em Saúde (BVS) em março de 2021. Essa base foi escolhida por conter fontes de informação nacionais e internacionais disponíveis na íntegra e de forma gratuita, como o Centro Latino-Americano e do Caribe de Informação em Ciências da Saúde (BIREME), Sistema Online de Busca e Análise de Literatura Médica (MEDLINE), Literatura Latino-Americana e do Caribe em Ciências da Saúde (LILACS) e Localizador de Informação em Saúde (LIS). 
Com o intuito de otimizar os resultados, foram realizadas 2 pesquisas em momentos diferentes na BVS, a primeira com os descritores "fertilidade" e "quimioterapia" e a segunda com os descritores "fertilidade" e "enfermagem". Foram utilizados os seguintes filtros na primeira pesquisa: texto disponível na íntegra, assunto principal "Preservação da Fertilidade; Fertilidade; Infertilidade Feminina; Neoplasias".

Já na segunda pesquisa, os filtros: texto disponível na íntegra, assunto principal "Enfermagem oncológica; Fertilidade; Saúde reprodutiva; Infertilidade; Preservação da fertilidade; Neoplasias; Fertilidade; Infertilidade feminina; Aconselhamento". Em ambas as pesquisas, limitou-se a artigos publicados nos últimos 5 anos. Foram excluídos estudos sobre fertilidade masculina e estudos cujo motivo da infertilidade não seja devido ao tratamento do câncer, assim como artigos de opinião e cartas editoriais. Para detalhamento do processo de busca, foram seguidas as orientações do Relatório Preferidos para Revisões Sistemáticas e Meta-Análises (PRISMA).

Todo o processo aconteceu em três etapas: a leitura dos títulos, seguida pela leitura dos resumos e a leitura integral. Essas etapas foram realizadas simultaneamente pelos pesquisadores, que compararam suas seleções e discutiram as divergências. Por fim, foram extraídos os resultados e conclusões mais relevantes dos estudos para compilação dos achados.

\section{RESULTADOS}

Foram localizados 286 artigos de acordo com a metodologia empregada. Após serem removidos os duplicados e indisponíveis, em associação com filtros e aplicação dos critérios de elegibilidade, restaram apenas 20 artigos para análise. Os detalhes da busca estão esquematizados no fluxograma da Figura 1.

Figura 1 - Fluxograma descrevendo a estratégia de busca e seleção de estudos dos itens Preferenciais de Relatórios para Revisões Sistemáticas e Meta-Análise (PRISMA).

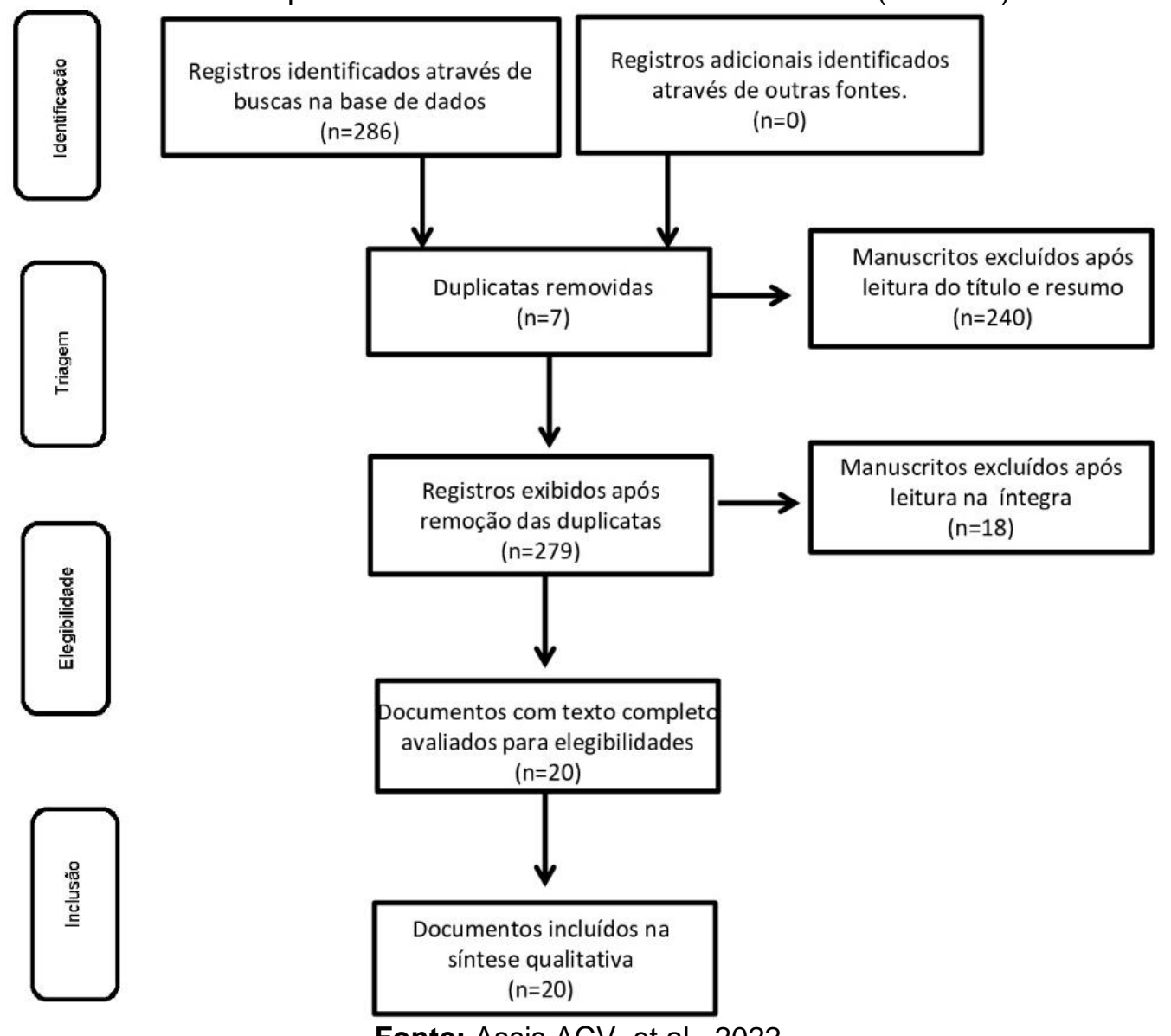

Fonte: Assis ACV, et al., 2022.

O Quadro 1 traz uma síntese dos principais achados nos estudos selecionados para este artigo, destacando trechos relacionados ao tratamento da fertilidade em mulheres em tratamento quimioterápico. 
Quadro 1 - Síntese dos principais achados relacionados ao tratamento da fertilidade em mulheres em tratamento quimioterápico.

\begin{tabular}{|c|c|c|}
\hline № & AUTORES (ANO) & PRINCIPAIS ACHADOS \\
\hline 1 & Walter JR, et al. (2016). & $\begin{array}{l}\text { El. Oncofertilidade é um novo campo interdisciplinar que antecipa as necessidades reprodutivas de pacientes com câncer que enfrentam } \\
\text { tratamento e cirurgia potencialmente ameaçadores da fertilidade. }\end{array}$ \\
\hline 2 & $\begin{array}{l}\text { Rodriguez WKA, et al. } \\
\text { (2017). }\end{array}$ & $\begin{array}{l}\text { El. A infertilidade induzida pelo tratamento do câncer é um problema reconhecido de sobreviventes, e a prática de PF está se espalhando } \\
\text { rapidamente. Os métodos clínicos atualmente estabelecidos de PF feminina incluem a criopreservação de embriões e oócitos maduros } \\
\text { obtidos após estimulação hormonal, pois a capacidade de descongelá-los e usá-los com sucesso em tratamentos de fertilidade foi } \\
\text { demonstrada. }\end{array}$ \\
\hline 3 & Letoirneau JM, et al. (2017). & $\begin{array}{l}\text { EP. A quimioterapia para câncer de mama tem sido associada à infertilidade e à menopausa precoce. Esses efeitos prejudiciais à saúde } \\
\text { reprodutiva foram associados à diminuição da qualidade de vida entre os sobreviventes do câncer de mama. Ter a capacidade de discutir } \\
\text { o potencial reprodutivo futuro de alguém antes da quimioterapia, bem como a capacidade de congelar oócitos ou embriões para uso futuro, } \\
\text { tem sido associada a melhorias na qualidade de vida. }\end{array}$ \\
\hline 4 & Chung JP, et al. (2017). & $\begin{array}{l}\text { EP. A PF ganhou atenção crescente em todo o mundo, à medida que os avanços no tratamento resultam em cada vez mais sobreviventes } \\
\text { que devem levar uma vida normal e começar uma família própria. Os avanços atuais na tecnologia reprodutiva permitiram que a fertilidade } \\
\text { fosse mantida pela PF apesar dos danos causados pelo TA. }\end{array}$ \\
\hline 5 & Villareal GC, et al. (2017). & $\begin{array}{l}\text { ET. Programas como "Jovens e Fortes: Programa de Cuidados e Pesquisa de Mulheres Jovens com Câncer de Mama", são dedicados } \\
\text { para mulheres jovens com câncer de mama, que podem melhorar a frequência de discussões sobre fertilidade e melhorias de preservação, } \\
\text { permitindo a entrega de informações padronizadas aos pacientes, promovendo alianças locais entre oncologia e centros de reprodução e } \\
\text { abordando sistematicamente barreiras, incluindo custos para pacientes carentes. }\end{array}$ \\
\hline 6 & Lambertini M, et al. (2018). & $\begin{array}{l}\text { EI. A IOP e a infertilidade subsequente são possíveis consequências adicionais do uso de quimioterapia em pacientes na pré-menopausa } \\
\text { com câncer de mama, com implicações negativas na qualidade de vida e sobrecarga psicológica. O aconselhamento sobre a oncofertilidade } \\
\text { é necessário para informar os pacientes sobre o possível desenvolvimento dos efeitos colaterais. A oferta do tratamento para PF deve ser } \\
\text { considerada. }\end{array}$ \\
\hline 7 & Dolmans MM, et al. (2018). & $\begin{array}{l}\text { RS. A maioria dos tratamentos de câncer, como quimioterapia, radioterapia ou uma combinação de ambos, são altamente tóxicos para as } \\
\text { gônadas, colocando meninas e mulheres jovens em risco de IOP e subsequente infertilidade. O aconselhamento sobre PF deve, portanto, } \\
\text { ser oferecido a todas as pacientes que necessitam de TA e àquelas que desejam adiar a maternidade por razões sociais / pessoais. }\end{array}$ \\
\hline 8 & Benoit A, et al. (2019). & $\begin{array}{l}\text { ECR. Algumas técnicas de PF aumentam a probabilidade de as sobreviventes se tornarem mães genéticas. As opções atuais de PF incluem } \\
\text { tratamentos médicos para proteção ovariana, criopreservação de oócitos e / ou embriões após estimulação ovariana controlada ou após } \\
\text { maturação in vitro e criopreservação de tecido ovariano, além de aconselhamento sobre oncofertilidade. }\end{array}$ \\
\hline 9 & Van Kol KGG, et al. (2019). & $\begin{array}{l}\text { RS. Para mulheres com câncer cervical em estágio IB2 que desejam preservar a fertilidade. Existem duas abordagens oferecidas. Opção } \\
\text { 1: QNBP para reduzir o tamanho do tumor para } 2 \mathrm{~cm} \text {. Opção } 2: \text { traquelectomia radical abdominal. Atualmente, permanece desconhecido } \\
\text { qual desses procedimentos tem os melhores resultados oncológicos e de fertilidade. }\end{array}$ \\
\hline 10 & Mikus M, et al. (2020). & $\begin{array}{l}\text { ER. Os avanços nas abordagens cirúrgicas tornaram a malignidade curável, com altas taxas de PF. Hoje em dia, a CPF com ou sem QA é } \\
\text { amplamente aceita devido ao alto sucesso do tratamento na doença em estágio inicial e pode, após discussão apropriada e consentimento } \\
\text { obtido, ser aplicada em mulheres jovens com TMCGO em estágio avançado que desejam gravidez. }\end{array}$ \\
\hline
\end{tabular}




\begin{tabular}{|c|c|c|}
\hline № & AUTORES (ANO) & PRINCIPAIS ACHADOS \\
\hline 11 & Policiano C, et al. (2020). & $\begin{array}{l}\text { ER. Pacientes com Linfoma de Hodgkin (um tipo de câncer que se origina no sistema linfático), tem como padrão ouro para tratamento a } \\
\text { quimioterapia nomeada ABVD. É considerado um tratamento de baixo risco gonadotóxico, porém, } 15 \text { a } 20 \% \text { dos pacientes recaem e } \\
\text { necessitam realizar a TCTH, um tratamento muito mais agressivo que causa falência ovariana prematura em uma alta proporção de } \\
\text { pacientes. Após o tratamento com quimioterapia, a recuperação dos ciclos menstruais normais não garante a fertilidade normal e RO, por } \\
\text { isso é necessário acompanhamento antes, durante e depois para a PF. }\end{array}$ \\
\hline 12 & $\begin{array}{l}\text { Mandelbaum RS, et al. } \\
\text { (2020). }\end{array}$ & $\begin{array}{l}\text { ENC. A demanda por PF está aumentando à medida que mais mulheres estão adiando o início da gravidez para a quarta e até a quinta } \\
\text { década de vida. Os avanços na ultrassonografia e o uso de níveis séricos de marcadores tumorais estão permitindo a detecção precoce de } \\
\text { malignidade ovariana, o que pode permitir a consideração das metas de fertilidade. }\end{array}$ \\
\hline 13 & Silvestris E, et al. (2020). & $\begin{array}{l}\text { RS. Sabe-se que o risco de infertilidade se correlaciona fortemente com o uso de AA (agentes com capacidade de se ligar ao DNA e causar } \\
\text { morte celular) e com a intensidade da quimioterapia, e para pacientes do sexo feminino também com a idade. A quimioterapia pode } \\
\text { prejudicar a OR de mulheres jovens em diferentes porcentagens. }\end{array}$ \\
\hline 14 & Patel P, et al. (2020). & $\begin{array}{l}\text { ET. A Sociedade Americana de Medicina Reprodutiva e a SAOC recomendam que os médicos que cuidam de pacientes adultos e } \\
\text { pediátricos com câncer de diferentes tipos discutam a PF antes do início da terapia anticâncer. Enquanto a quimioterapia e a radioterapia } \\
\text { são eficazes na eliminação das células cancerosas, os efeitos adversos no sistema reprodutivo persistem. }\end{array}$ \\
\hline 15 & D'hondt C, et al. (2020). & $\begin{array}{l}\text { EC. Pacientes com câncer de mama diagnosticados entre janeiro de } 2012 \text { e dezembro de } 2017 \text { submetidos a PF em um centro acadêmico } \\
\text { de fertilidade terciário antes da QA provam que a PF não atrasa o início da quimioterapia em pacientes com câncer de mama. }\end{array}$ \\
\hline 16 & Aljaser F, et al. (2020). & $\begin{array}{l}\text { EP. A PF pode envolver proteção ovariana entre outros métodos. No entanto, para resultados ideais, sugere-se que a seleção da estratégia } \\
\text { de preservação deve ser individualizada e projetada com base na avaliação de risco de fertilidade para cada caso. Recomenda-se que os } \\
\text { pacientes sejam bem informados o mais cedo possível, com tempo suficiente antes do tratamento, a fim de fazer uma escolha clara da } \\
\text { abordagem de preservação. }\end{array}$ \\
\hline 17 & Violette C, et al. (2021). & $\begin{array}{l}\text { RS. São necessários esforços para melhorar o aconselhamento pré-operatório, aumentar a criopreservação de oócitos e avaliar a } \\
\text { segurança da preservação dos órgãos reprodutivos no momento da CPF. }\end{array}$ \\
\hline 18 & Karavani G, et al. (2021). & $\begin{array}{l}\text { EC. O risco geral de IO em pacientes submetidas ao TG foi de } 30 \% \text { após um seguimento médio de } 50 \text { meses. As diretrizes defendem o } \\
\text { aconselhamento de pacientes jovens com câncer sobre o possível efeito gonadotóxico da quimioterapia e as opções disponíveis para PF. } \\
\text { As opções sugeridas para PF em mulheres incluem a criopreservação de oócitos, embriões e tecido ovariano. }\end{array}$ \\
\hline 19 & Zamani N, et al. (2021). & $\begin{array}{l}\text { ER. Pacientes com TMCGO podem engravidar e dar a luz se desejarem. O estágio avançado do tumor não foi o fator convincente para } \\
\text { evitar a PF. A CPF é um tratamento seguro e resulta em uma alta taxa de fertilidade. }\end{array}$ \\
\hline 20 & Kasius JC, et al. (2021). & $\begin{array}{l}\text { RS. A QN tem se mostrado eficaz na redução do tamanho do tumor no CE. Em pacientes que desejam preservar a fertilidade, a QN pode } \\
\text { ser usada para diminuir o tamanho do tumor e, subsequentemente, prosseguir com a cirurgia de PF. }\end{array}$ \\
\hline \multicolumn{3}{|c|}{$\begin{array}{l}\text { Legenda: PF: Preservação da Fertilidade. TG: Tratamento Gonadotóxico. IOP: Insuficiência Ovariana Prematura. IO: Insuficiência Ovariana. El: Estudo de Incidência. RS: Revisão } \\
\text { Sistemática. AA: Agentes Alquilantes. RI: Revisão Integrativa. ECR: Estudo de Coorte Retrospectivo. SAOC: Sociedade Americana de Medicina Reprodutiva. ECC: Ensaio Clínico } \\
\text { Controlado. IB2: O tumor tem entre } 2 \text { e } 4 \mathrm{~cm} \text { de tamanho, mas não se espalhou para os linfonodos próximos. QNBP: Quimioterapia Neoadjuvante à Base de Platina. EC: Estudo } \\
\text { de Caso. ER: Estudo Retrospectivo. TMCGO: Tumor Maligno de Células Germinativas do Ovário. CPF: Cirurgia de Preservação da Fertilidade. ABVD:Adrimacina, Bleomicina, } \\
\text { Vimblastina, Docarbazina. TCTH: Transplante de Células Tronco Hematopoéticas. ENC: Ensaio Clínico. CEO: Câncer Epitelial de Ovário. RO: Reserva Ovariana. ET: Estudo } \\
\text { Transversal. QN: Quimioterapia Neoadjuvante. QA: Quimioterapia Adjuvante. EP: Estudo de Prognóstico. CC: Cirurgia Citorredutora. QIH: Quimioterapia Intraperitoneal } \\
\text { Hipertérmica. CE: Câncer Endometrial. HEA: Hiperplasia Endometrial Atípica. EOR: Estudo Observacional Retrospectivo. Fonte: Assis ACV, et al., 2022. }\end{array}$} \\
\hline
\end{tabular}




\section{DISCUSSÃO}

Câncer, de acordo com o INCA, é um termo que se relaciona a 100 diferentes tipos de doenças malignas que têm em comum o crescimento descontrolado das células, que podem invadir os tecidos adjacentes ou órgãos distantes. Estas células têm a tendência de se dividir de forma rápida, agressiva e incontrolável, levando a formação de um ou vários tumores, que espalham-se por todo o corpo. As causas do câncer podem ser variadas e são classificadas em externas ou internas, estando ambas relacionadas umas às outras (INCA, 2020c). As causas externas estão ligadas ao meio ambiente e aos hábitos de vida de cada indivíduo. Já as causas internas, são, na maioria dos casos, genéticas, relacionando-se à eficácia do organismo de combater as agressões externas. Dos casos de cânceres, 80 a $90 \%$ estão ligados a fatores ambientais. O câncer pode surgir dependendo da intensidade e do tempo de exposição aos fatores que o provocam (INCA, 2020a).

Na medicina, existe uma especialidade que é a oncofertilidade e, de acordo com a Sociedade Brasileira de Reprodução Assistida (SBRA), esta especialidade tem como principal objetivo preservar a fertilidade de pacientes oncológicos que pretendem, após o tratamento, ter filhos. Walter JR, et al. (2016), trazem em seu estudo a oncofertilidade como um novo campo interdisciplinar que antecipa as necessidades reprodutivas de pacientes na oncologia. Já D'Hondt C, et al. (2020), trata a oncofertilidade como um campo interdisciplinar que une ciências sociais e a biomédicas e examina questões relacionadas às opções de fertilidade de um indivíduo, escolha e objetivos à luz do diagnóstico de câncer, tratamento e sobrevivência.

Para Lambertini M, et al. (2018), a oncofertilidade é necessária para informar aos pacientes sobre o possível desenvolvimento dos efeitos colaterais, além de considerar a oferta do tratamento para a preservação da fertilidade. A Sociedade Americana de Medicina Reprodutiva (SAMR) e a Sociedade Americana de Oncologia Clínica (SAOC) recomendam que os médicos que cuidam de pacientes adultos e pediátricos com câncer discutam sobre a preservação da fertilidade antes do início da terapia anticâncer (PATEL P, et al., 2020).

O tratamento do câncer é feito por uma ou várias técnicas de tratamento combinadas. A principal delas é a cirurgia oncológica, que pode ser combinada com o transplante de medula óssea, com a radioterapia ou com a quimioterapia. O transplante de medula óssea é o tratamento utilizado para algumas doenças malignas que afetam as células do sangue, como a leucemia, onde é feita a substituição da medula óssea do paciente doente por células saudáveis de uma outra medula óssea. Já a radioterapia é um tipo de tratamento onde as radiações são utilizadas para impedir que as células de um tumor se desenvolvam ou, até mesmo, para tentar destruir esse tumor. Outro tipo de tratamento é a quimioterapia que utiliza medicamentos farmacológicos para tentar impedir a proliferação de células anormais e, até mesmo, a formação de um tumor (MINISTÉRIO DA SAÚDE, 2019).

O tratamento quimioterápico pode ser classificado em adjuvante ou neoadjuvante. A quimioterapia adjuvante é realizada após a cirurgia para eliminar micro metástases e é usada para casos iniciais de câncer, reduzindo a recorrência da doença e a mortalidade dos pacientes. Já a quimioterapia neoadjuvante é aplicada antes da cirurgia para reduzir o tumor ou para permitir cirurgias conservadoras (COELHO RCFP, et al., 2019).

No estudo de Kasius JC, et al. (2021), a técnica neoadjuvante tem se mostrado eficaz na redução do tamanho do tumor no câncer cervical. Em pacientes que desejam preservar a fertilidade, pode ser usada para diminuir o tamanho do tumor e, subsequentemente, prosseguir com a cirurgia de preservação da fertilidade. Mikus M, et al. (2020) e Zamani N, et al. (2021), descrevem que hoje em dia a Cirurgia de Preservação da Fertilidade (CPF), com ou sem quimioterapia adjuvante, é amplamente aceita devido ao alto sucesso do tratamento na doença em estágio inicial; pode ser aplicada em mulheres jovens com Tumor Maligno de Células Germinativas do Ovário (TMCGO) em estágio avançado que desejam engravidar, pois o estágio avançado do tumor não foi fator convincente para evitar o tratamento da preservação da fertilidade.

Segundo Dolmans MM, et al. (2018), a maioria dos tratamentos de câncer são altamente tóxicos para as gônadas, colocando meninas e mulheres jovens em risco de IOP e subsequente infertilidade, alterando negativamente a vida das mulheres com impactos físicos, emocionais, econômicos e sociais. Silvestris $E$, et al. (2020) e Van Kol KGG, et al. (2019), relataram em seus estudos que o risco de infertilidade se correlaciona fortemente com o uso de agentes alquilantes (agentes com capacidade de se ligar ao DNA e causar morte celular), como a ifosfamida e a cisplatina, que são prejudiciais aos folículos ovarianos e estão associados à 
fibrose ovariana e podem levar à IOP. Porém, Policiano C, et al. (2020), trazem que a quimioterapia nomeada Adrimacina, Bleomicina, Vimblastina, Docarbazina (ABVD) é considerada um tratamento de baixo risco gonadotóxico.

A Preservação da Fertilidade (PF) é frequentemente possível e deve ser abordada de forma precoce, uma vez que, quanto maior o tempo disponível antes do início da quimioterapia, maior será a probabilidade de sucesso [38\%]. Chung JP, et al. (2017), relata que, se a fertilidade puder ser preservada antes do tratamento, maior será a capacidade de manter ou recuperar a fertilidade após o término do tratamento. Há, no Brasil, legislações específicas que definem e regem a reprodução assistida como a Resolução no 2.294 de 27 de maio de 2021 do Conselho Federal de Medicina, o Código de Ética Médica e a Lei no 11.105/05, denominada Lei de Biossegurança. A lei traz que, todo cidadão brasileiro tem o direito à informação é a utilização das técnicas de reprodução assistida para ter filho ou preservar a fertilidade, isso inclui pacientes diagnosticados com câncer que iniciaram o tratamento oncológico, obrigando a equipe multidisciplinar a informar, de forma clara os efeitos e sucessos desses métodos.

Benoit A, et al., (2020), relatam que a PF pode envolver proteção ovariana entre outras técnicas que aumentam a probabilidade das sobreviventes se tornarem mães genéticas. Os métodos de PF incluem a cirurgia de preservação da fertilidade, onde é realizada a retirada de um dos ovários que é afetado pelo câncer, e agonistas do hormônio liberador de gonadotrofina para supressão gonadal durante a quimioterapia. Além disso, a tecnologia de reprodução assistida, expandiram as opções de PF que agora podem ser aplicadas a um espectro mais amplo de pacientes, incluindo aquelas que estão na pré-puberdade e também aquelas com tempo insuficiente antes do início do tratamento (CHUNG JP, et al., 2017).

Ademais, as opções atuais de PF incluem a criopreservação de tecido ovariano, a criopreservação de oócitos (óvulos), a criopreservação de embriões (fertilização in vitro), a traquelectomia radical (retirada do colo do útero), a terapia com progesterona, além do aconselhamento sobre oncofertilidade. A criopreservação de tecido ovariano é a retirada do ovário, inteiro ou parte dele, por um procedimento denominado de laparoscopia, onde o médico faz uma pequena incisão perto do umbigo da paciente e introduz um tubo fino e flexível. Após a retirada, o ovário é dividido em pequenas tiras que são congeladas e armazenadas para uso futuro. A criopreservação de oócitos (óvulos) é a remoção de óvulos maduros os quais são congelados antes de serem fertilizados (ALJASER F, 2020).

A criopreservação de embriões (fertilização in vitro) se dá quando os óvulos retirados da mulher são fertilizados em laboratório e os embriões gerados por essa fertilização são congelados e utilizados após o tratamento do câncer, a fim de que a mulher possa gerar os próprios filhos. Já a traquelectomia radical é o procedimento, durante a cirurgia para tratar o câncer do colo do útero, onde o médico remove por completo o colo doente, mas preserva o útero e também os ovários, e realiza uma sutura ou aplica uma fita especial que possa agir como o colo retirado. Uma pequena abertura nesta sutura ou nesta fita permite que a mulher possa menstruar normalmente, assim como permite também que o esperma chegue ao útero e às trompas para poder fertilizar um óvulo. Por meio de um dispositivo intrauterino ou até mesmo com o uso de pílulas, é realizada a terapia com progesterona em mulheres com câncer de endométrio estágio 1, grau 1 e que ainda desejam ter filhos (ALJASER F, 2020).

Villarreal-Garza C, et al. (2017); Violette C, et al. (2021) e Karavani G, et al. (2021), relataram que políticas e diretrizes no âmbito do Sistema Único de Saúde (SUS) defendem o aconselhamento de pacientes jovens com câncer sobre o possível efeito gonadotóxico da quimioterapia e as opções disponíveis para PF. São necessários esforços para melhorar o aconselhamento a essas pacientes, ofertando programas que são dedicados para as mulheres jovens com câncer que podem melhorar a frequência das discussões sobre PF, o que permite a entrega de informações padronizadas a essas pacientes, promovendo alianças locais entre a oncologia e os centros de reprodução e também abordar sistematicamente barreiras, incluindo custos para pacientes carentes.

No que diz respeito à seleção da estratégia de preservação, Aljaser $F(2020)$, relata que ela deve ser individualizada e projetada com base na avaliação de risco de fertilidade para cada caso. Para seu sucesso, requer a colaboração entre oncologistas, ginecologistas, tecnólogos de PF, outros profissionais de saúde e, mais importante, a do próprio paciente. Recomenda-se que os pacientes sejam bem informados o mais cedo 
possível, com tempo suficiente antes do tratamento, a fim de fazer uma escolha clara da abordagem de preservação. Nesse contexto, é necessária uma abordagem multidisciplinar para o melhor acompanhamento da mulher no decorrer de todas as etapas, seja no tratamento ou na busca pela fertilidade. No entanto, a equipe de enfermagem tem se destacado.

Conforme o Artigo 11, da Lei no 7.498 de 25 de junho de 1986, com relação às atividades e atribuições dos Enfermeiros, compete a este, privativamente todos os cuidados diretos aos pacientes com gravidade que gere risco de morte, executar intervenções complexas, que necessite de entendimento científico, e a aptidão em resolver questões de forma rápida. Além disso, de acordo com a Resolução do Conselho Federal de Enfermagem (COFEN) (1998), é de competência do Enfermeiro que trabalha com quimioterápico antineoplásico administrá-lo, elaborar protocolos para prevenção, tratamento e diminuição de efeitos colaterais nos pacientes em uso de quimioterapia, além de proporcionar ações de prevenção de riscos e agravos, através da educação dos pacientes e familiares, a fim de garantir maior qualidade de vida para o paciente.

Estudos internacionais mostram o impacto positivo da enfermagem na prática avançada, levando a melhoria dos resultados aos pacientes, na qualidade da assistência e nos obstáculos que os sistemas de saúde apresentam. Atualmente, mais de 70 países estão interessados em implantar a prática avançada em enfermagem (SCHNEIDER F, et al., 2021). De todos os profissionais da equipe multidisciplinar na saúde, a enfermagem tem uma função primordial para lidar com o enfrentamento de uma pessoa que e portadora de câncer, utilizando estratégias que visam amenizar o desconforto emocional e físico, a fim de promover a qualidade de vida. A oncologia é uma área que requer uma alta demanda da assistência da enfermagem, que se destaca por atuar de forma empática e afetiva. Com isso, os profissionais dessa área, têm o contato direto com a dor e reações inesperadas no processo de desesperança do paciente. (MACEDO A, et al., 2019).

A estimativa mundial mostra que, no ano de, 2018, ocorreram 18,1 milhões de casos novos de câncer e 9,6 milhões de óbitos. Estima-se que para o Brasil, de 2020 a 2022, haverá 625 mil casos novos de câncer a cada ano. Levando em consideração esses dados, é evidente a importância de profissionais, com conhecimentos, habilidades e atitudes específicas para atuar frente às necessidades complexas destes pacientes, destacando-se, assim, a implantação da Enfermagem de Prática Avançada (EPA) no cenário da oncologia (SCHNEIDER F, et al., 2021).

\section{CONSIDERAÇÕES FINAIS}

Com esse estudo, foi possível observar que os diversos tratamentos para o câncer, em especial a radioterapia e a quimioterapia, têm uma ação altamente tóxica para as gônadas, os quais colocam as mulheres em idade reprodutiva em risco de desenvolver infertilidade. A preservação da fertilidade é algo possível e que engloba a necessidade de se abordar, aconselhar, informar e discutir a probabilidade de reprodução futura das pacientes antes do tratamento de quimioterapia, bem como a escolha do método de preservação para uso futuro, aumentando, assim, a possibilidade de as sobreviventes se tornarem mães. $O$ tratamento do câncer requer uma abordagem multidisciplinar para o melhor acompanhamento no decorrer de todas as etapas. Porém, a equipe de enfermagem destaca-se por abordar o paciente como um todo e não focando somente na doença, oferecendo apoio, aconselhamento e educação em saúde no que diz respeito à preservação da fertilidade, a fim de amenizar os impactos psicológicos, sociais e emocionais no decorrer do tratamento.

\section{REFERÊNCIAS}

1. ALJASER F. Preservation of fertility in female: Indications, available options, and current status in Saudi Arabia. Seminars in Oncology, 2020; 47(6): 390-7.

2. ASHIZAWA M, KANDA Y. Preservation of fertility in patients with hematological malignancies. Japanese Journal of Clinical Oncology, 2020; 50(7): 729-42.

3. BENOIT A, et al. Does a web-based decision aid improve informed choice for fertility preservation in women with breast cancer? Study protocol for a randomised controlled trial. BMJ Open, 2020;10(2): e031739.

4. CARMO RALO, et al. Cuidar em Oncologia: Desafios e Superações Cotidianas Vivenciados por Enfermeiros. Revista Brasileira de Cancerologia, 2019; 65(3).

5. CHUNG JP, et al. Evaluation of the awareness of, attitude to, and knowledge about fertility preservation in cancer patients among clinical practitioners in Hong Kong. Hong Kong Med. J.,2017; 23(6): 556-61. 
6. COELHO RCFP, et al. Tratamento quimioterápico adjuvante e neoadjuvante e as implicações na qualidade de vida mulheres com câncer de mama. Revista de Enfermagem da Universidade Federal de Pernambuco (UFPE) ,(Supl. 11)2017: 4732-40.

7. D'HONDT C, et al. Fertility preservation does not delay the initiation of chemotherapy in breast cancer patients treated with adjuvant or neoadjuvant chemotherapy. Breast Cancer Res. Treat., 2020; 184(2): 433-44.

8. DOLMANS M-M, MANAVELLA DD. Recent advances in fertility preservation: Advances in fertility preservation. J. Obstet. Gynaecol. Res., 2019; 45(2): 266-79.

9. INSTITUTO NACIONAL DE CÂNCER (INCA). Estatísticas de câncer. 2020a. Disponível em: https://www.inca.gov.br/numeros-de-cancer. Acessado em: 22 de mar. de 2021.

10. INSTITUTO NACIONAL DE CÂNCER (INCA). Estimativa de câncer em 2020. 2021b. Disponível em: https://www.inca.gov.br/estimativa/introducao. Acessado em: 22 de mar. de 2021.

11. INSTITUTO NACIONAL DE CÂNCER (INCA). O que é câncer. 2020c. Disponível em: https://www.inca.gov.br/o-quee-cancer. Acessado em: 19 de mai. de 2021.

12. INSTITUTO NACIONAL DE CÂNCER (INCA). Tratamento do Câncer: Quimioterapia. 2021d. Disponível em: https://www.inca.qov.br/tratamento/quimioterapia. Acessado em: 22 de mar. de 2021.

13. JAYASINGHE YL, et al. Ovarian function, fertility and reproductive lifespan in cancer patients. Expert Review of Endocrinology \& Metabolism.,2018; 13(3):125-36.

14. KARAVANI G, et al. Chemotherapy-based gonadotoxicity risk evaluation as a predictor of reproductive outcomes in post-pubertal patients following ovarian tissue cryopreservation. Biomed Central Ltd (BMC) Women's Health, 2021; 21(1): 280.

15. KEIM-MALPASS J, et al. What is the Role of the Oncology Nurse in Fertility Preservation Counseling and Education for Young Patients?. Journal of Cancer Education, 2018; 33(6):1301-5.

16. LAMBERTINI M, et al. Prospective study to optimize care and improve knowledge on ovarian function and/or fertility preservation in young breast cancer patients: Results of the pilot phase of the Pregnancy and Fertility (PREFER) study. The Breast, 2018; 41:51-6.

17. LETOURNEAU JM, et al. Random start ovarian stimulation for fertility preservation appears unlikely to delay initiation of neoadjuvant chemotherapy for breast cancer. Human Reproduction, 2017; 32(10):2123-9.

18. MACEDO A, et al. Estratégias de enfrentamento dos profissionais de enfermagem frente à morte na oncologia pediátrica: revisão integrativa. Rev. pesqui. cuid. fundam. 2019; 718-724.

19. MANDELBAUM RS, et al. Fertility-sparing treatment for early-stage epithelial ovarian cancer: Contemporary oncologic, reproductive and endocrinologic perspectives. Journal of Obstetrics and Gynaecology Research, 2020; 46(8):126381.

20. MIKUŠ M, et al. Fertility-sparing surgery for patients with malignant ovarian germ cell tumors: 10 years of clinical experience from a tertiary, referral center. Archives of Gynecology and Obstetrics, 2020; 301(5):1227-33.

21. MINISTÉRIO DA SAÚDE. Câncer: sintomas, causas, tipos e tratamentos. 2020. Disponível em: http://antigo.saude.gov.br/saude-de-a-z/cancer. Acessado em: 6 de nov. de 2021.

22. MORETTO IG, et al. Acompanhamento por telefone como intervenção de enfermagem a pacientes em quimioterapia ambulatorial: revisão integrativa. Revista Gaúcha Enfermagem, 2019; 40: e20190039.

23. OLIVEIRA FPB, GOMES KR. O enfermeiro diante do problema de infertilidade: uma abordagem de enfrentamento. Repositório Institucional Faculdade de Educacãa e Meio Ambiente (FAEMA), 2019.

24. PATEL P, et al. Evaluation of Reported Fertility Preservation Counseling Before Chemotherapy Using the Quality Oncology Practice Initiative Survey. JAMA Network Open, 2020; 3(7): e2010806.

25. POLICIANO C, et al. Impact of ABVD chemotherapy on ovarian reserve after fertility preservation in reproductive-aged women with Hodgkin lymphoma. Journal of Assisted Reproduction and Genetics, 2020; 37(7):1755-61.

26. QUEIROZ ABA, et al. Nursing work in assisted human reproduction: between technology and humanization. Revista Brasileira de Enfermagem, 2020; 73(3).

27. RASHEDI AS, et al. Survey of Fertility Preservation Options Available to Patients With Cancer Around the Globe. Journal of Global Oncology (JGO), 2020; (6):331-44.

28. RODRIGUEZ WKA, et al. Safety of fertility preservation in breast cancer patients in a register-based matched cohort study. Breast Cancer Research and Treatment, 2018; 167(3):761-9.

29. SCHNEIDER F, et al. Training of advanced practice nurses in oncology for the best care: a systematic review. Revista da Escola de Enfermagem da Universidade de São Paulo (USP), 2021; 55: e03700.

30. SILVA L, et al. Perfil epidemiológico de pacientes oncológicos atendidos em um hospital de referência da região centrooeste do Brasil. Enciclopédia Biosfera, 2019; 16(29):2106-19.

31. Silva S, et al. Fertilidade e contracepção em mulheres com câncer em tratamento quimioterápico. Escola Anna Nery, 2021; 25(1): e20190374.

32. SILVESTRIS E, et al. Novel aspects on gonadotoxicity and fertility preservation in lymphoproliferative neoplasms. Critical Reviews in Oncology/Hematology, 2020; 151:102981.

33. TOMÁS C, et al. Preservação da fertilidade em doentes oncológicos ou sob terapêutica gonadotóxica: estado da arte. Reprodução \& Climatério, 2016; 31(1):55-61.

34. VAN KOL KGG, et al. Abdominal radical trachelectomy versus chemotherapy followed by vaginal radical trachelectomy in stage 1B2 (FIGO 2018) cervical cancer. A systematic review on fertility and recurrence rates. Gynecologic Oncology, $2019 ; 155(3): 515-21$.

35. VILLARREAL-GARZA C, et al. Fertility concerns among breast cancer patients in Mexico. The Breast, 2017; 33:71-5.

36. VIOLETTE C, et al. Fertility after cytoreductive surgery and hyperthermic intraperitoneal chemotherapy: A call to action. Journal of Surgical Oncology, 2021; 123(4):1045-9.

37. WALTER JR, et al. Oncofertility considerations in adolescents and young adults given a diagnosis of melanoma: Fertility risk of Food and Drug Administration approved systemic therapies. Journal of the American Academy of Dermatology, 2016.

38. ZAMANI N, et al. Fertility sparing surgery in malignant ovarian Germ cell tumor (MOGCT): 15 years experience. Biomed Central Ltd (BMC) Women's Health, 2021; 21(1): 282.

39. KASIUS JC, et al. Neo-adjuvant chemotherapy in fertility-sparing cervical cancer treatment. Best Practice \& Research Clinical Obstetrics \& Gynaecology, 2021; 75:82-100.

40. ROO SF, et al. Global oncofertility index data gap slows progress. Biology of Reproduction, 2017; 96(6):1124-8. 